\title{
Literatur
}

1. Grassmann, W., K. Hannig und M. Knedel, Dtsch. Med. Wschr. 76, 333 (1951). GrassmaNn, W., Naturwissenschaften 38, 200 (1951). - 2. Pezold, F. A. und U. Peiser, Klin. Wschr. 31, 982 (1953). - 3. Konn, J., Arztl. Lab. 10, 223 (1964). Arztl. Lab. 10, 269 (1964). - 4. Meulemans, O., Clin. chim. Acta Amsterdam 3, 275 (1958). - 5. Mrckr, H., Mh. Chem. 83, 210 (1956). - 6. Busse, V., in Vorbereitung. - 7. Deutsche Forschungsgemeinschaft, Farbstoffkommission 6. u. 12. Mitt. Fr. Steiner-Verlag Wiesbaden (1957) und (1965). - 8. BARrollrer, J., E. WAtZke und H. Gibian, Z. Naturforsch. 136, 754 (1958). 9. Sadini, V., Rass. Chim. 12, 17 (1960). - 10. Gänshirt, H., D. WALDI und E. StahL, in: Dünnschichtchromatographie, S. 360, Hrsg. Stahl, E., Springer-Vërlag Berlin (1962). - 11. Wollenweber, P., J. Chromatogr. 7, 557 (1962). - 12. Verma, M. R. und R. DAs, J. Sci. Ind. Res. 15c, 186 (1956). - 13. SRAMEK, J., Dt. Textiltechn. 13, 594 (1963). - 14. KolsEK, J., Chemiker-
Ztg. 82, 35 (1958). - 15. Odebrecht, W., Arzn. Standard. 4, 271 (1963). - 16. GrassmanN, W. und L. HüBNER, Naturwissenschaften 40, 272 (1953). - 17. GrASSMANN, W. und I. HüBNER, Leder 5, 49 (1954). - 18. AbDeL-Wahab, E. M. und D. J. R. Laurence, Biochem. J. 60, XXXV (1955). - 19. Schultz, G., Farbstofftab., 7. Aufl., 1. Band Akadem. Verlagsges. m.b.H., Leipzig (1931). - 20. Colour-INDEx, publ. by Soc. of Dyers and Colourist. 2. Aufl.; Bredford; Soc. of Dyers and Colourists (1956-1963). - 21. Harms, H., Handbuch der Farbstoffe für die Mikroskopie, Staufen-Verl. Kamp-Lintfort (1957). - 22. SRAMEK, J., J. Chromatogr. 15, 57 (1964). - 23. Zit. nach HARMS, in Stain Techn. 10, 73 (1953). - 24. Kawerau, E. Analyst 79, 681 (1954). 25. Farbstoffuntersuchung, in: Ullmanns Enzyklopädie der techn. Chemie. 3. Aufl. Bd. 7, S. 212, Urban und Schwarzenberg, Berlin (1956).
Dr. V. Busse

1000 Berlin 45

Klinikum der Freien Universität

\section{Bestimmung von Acetat in biologischem Material durch Gaschromatographie}

\author{
Von J. FRöHLICH${ }^{1}$ ) und O. WIELAND \\ Aus dem Klinisch-Chemischen Institut des Städtischen Krankenbauses München-Schwabing und der \\ Biochemischen Abteilung der Forschergruppe Diabetes (Leiter: Prof. Dr. O. Wieland)
}

(Eingegangen am 15. März 1968)

Herrn Prof. Dr. Dr. Ernst Schütte zum 60. Geburtstag gewidmet

\begin{abstract}
Es wird eine Methode zur gaschromatographischen Acetatbestimmung in biologischem Material nach vorausgehender Abtrennung der Essigsäure durch Mikrodestillation beschrieben. Die Bestimmung erfolgt auf einer etwa $160 \mathrm{~cm}$ langen Glassäule ( $\varnothing 4 \mathrm{~mm}) \mathrm{mit}$ FFAP2) als stationärer Phase auf Chromosorb WAW 80-100 mesh bei $180^{\circ}$. Es wird eine Trennstufenzahl von 1600 erreicht, die Trennleistung beträgt 1000. Die $C_{2}$-Bande erscheint vollständig vom Wassergịpfel getrennt ohne wesentliches Schweifen. Die Auswertung erfolgt durch Mitführen eines Inneren Propionsäurestandards und zusätzlich an Hand von Eichkurven. Bei der Bestimmung werden im Mittel $87 \%$ des eingesetzten Acetats wiedergefunden.
\end{abstract}

A method is reported for the gas chromatographic determination of acetate in biological material, following a preliminary separation of the acetic acid by microdistillation. The determination is performed in an approx. $160 \mathrm{~cm}$ long glass column ( $\varnothing \mathrm{mm})$ on Chromosorb WAW $80-100$ mesh at $180^{\circ}$ with FFAP ${ }^{2}$ ) as the stationary phase. A theoretical plate number of 1600 was achieved and the separation efficiency was 1000 . The band is completely separated from the water peak with essentially no tailing. The results are evaluated by the use of an internal standard of propionic acid and standard curves. On average, $87 \%$ of the acetate is recovered in the determination.

Acetat tritt in freier Form als obligates Stoffwechselprodukt bei Mensch und Tier auf. Seine Konzentration in. Körpersäfțen und Geweben ist im allgemeinen - mit Ausnahme von Pflanzenfressern - sehr gering, wodurch einer exakkten Analytik gew̌isse Grenzen gesetzt sind. Für die bisher auf dem Gebiet des Essigsäurestoffwechsels durchgeführten Arbeiten wurde zum größten Teil der von LundQurst (1) angegebene Sulfanilamid-Acetylierungstest verwendet. Hierbei wird die Essigsäure vorher durch Mikrodiffusion nach Conway (2) aus den enteiweißten Gewebsextrakten abgetrennt. Die praktische Anwendbarkeit anderer Verfahren zur Essigsäurebestimmung, wie einer optisch-enzymatischen (3) oder einer gaschromatographischen (4) Methode läßt sich mangels

1) Stipendiat der Deutschen Forschungsgemeinschaft.

2) s. Fußnote S. 2 ausreichender Erfahrungen bisher nicht mit Sicherheit beurteilen. In eigenen Versuchen schien uns der Sulfanilamid-Acetylierungstest noch immer am brauchbarsten, wobei wir einen Fortschritt in der Einführung der Mikrodestillation nach BARTLEY (5) an Stelle der Conway-Diffusion sehen.

Das Bedürfnis nach weiterer methodischer Verbesserung und Vereinfachung erwuchs in Zusammenhang mit ausgedehnten tierexperimentellen Untersuchungen über den Acetatstoffwechsel in Abhängigkeit von der Ernährung (6), sowie im Hinblick auf geplante Studien über das Verhalten der Essigsäure im Blut und anderen Körperflüssigkeiten beim Menschen in Abhängigkeit von verschiedenen Krankheiten. Die im folgenden beschriebene gaschromatographische Methode hat sich in unseren Händen als geeignet zur Lösung dieser Aufgabe erwiesen. 


\section{Methodik}

\section{Aufbereitung des Untersuchungsmaterials}

Blut

Es werden etwa $10 \mathrm{~m} l$ Blut aus der $V$. cubitalis in eisgekühlte Zentrifugengläschen entnommen, die vorher mit drei Tropfen Heparin (5000 I.E./ml, Novo Industrie) beschickt worden waren. Nach Zentrifugieren bei $+4^{\circ}$ (3000 U./Min.) wird das Plasma abpipettiert und mit dem halben Volumen 12proz. eiskalter Perchlorsäure enteiweißt. Nach erneutem Zentrifugieren (3000 U./ Min.) werden $3 \mathrm{~m} l$ des Überstandes mit $0,1 \mathrm{~m} l 0,04$ proz. wäßr. Thymolblaulösung als Indikator versetzt. Anschließend werden die Proben mit 6N Natronlauge auf $\mathrm{pH} 3$ eingestellt (Umschlag von Rot nach Gelb) und mit 0,5M Citratpuffer ( $\mathrm{pH} \mathrm{3}$ ) auf $4 \mathrm{ml}$ aufgefüllt. Für die folgende Mikrodestillation werden von den gepufferten Proben $2 \mathrm{mal}$ je $1,5 \mathrm{ml}$ in Mikrodestillationsgefäße nach BARTLEY (5) eingesetzt und diese anschließend evakuiert. Die Destillation erfolgt über $1 \frac{1}{2} \mathrm{Stdn}$. bei $55^{\circ}$ in auf $-10^{\circ}$ gekühlte Schliffgläser, in die $0,3 \mathrm{~m} / 0,1 \mathrm{~N}$ Natronlauge vorgelegt sind. Vom Destillat werden anschließend je $1,2 \mathrm{ml}$ in Spitzgläschen übertragen und im Glycerinbad bei $120^{\circ}$ langsam zur Trockene eingedampft. Die eingedampften Proben werden im Kühlschrank aufbewahrt und erst unmittelbar vor der gaschromatographischen Auswertung mit $0,05 \mathrm{ml}$ eisgekühlter $0,4 \mathrm{~N}$ Salzsäure aufgenommen, der Propionsäure (puriss. p. A., Fluka) in 0,5 mm Endkonzentration zugesetzt ist.

\section{Organe}

Organentnahme, Aufschluß und Perchlorsäureextraktion exfolgten wie an anderer Stelle beschrieben (7). Der sich mit der Pufferung der. Proben anschließende weitere Arbeitsgang entspricht dem unter „Blut" angegebenen.

\section{Andere Körperflüssigkeiten}

Die Behandlung eiweißhaltiger Proben entspricht der von Blutproben. Harn und Liquor cerebrospinalis werden direkt mit dem gleichen Volumen Citratpuffer auf $\mathrm{pH} 3$ eingestellt und anschlieBend zur Mikrodestillation eingesetzt. Eine vorausgehende Enteiweißung ist hier unter normalen Bedingungen nicht erforderlich.

\section{Eichlösungen und Blindwert}

Ausgehend von einer $10 \mathrm{~mm}$ Natriumacetatstammlösung (Natriumacetat krist. p. A., Merck), die im Kühlschrank gut verschlossen aufbewahrt wird, wird am Versuchstage eine Verdünnungsreihe hergestellt, die wie die Proben mit Perchlorsäure behandelt und auf $\mathrm{pH} 3$ eingestellt wird. Als Blindwert dient eine Lösung gleicher Zusammensetzung, jedoch ohne Acetatzusatz.

\section{Gaschromatographie}

\section{Gerät}

Die Messungen werden mit dem Gaschromatographen der Fa. Packard Nr. 7821 durchgeführt, der mit einem Flammenionisationsdetektor (FID) ausgestattet ist. Die Trennung erfolgt auf einer etwa $160 \mathrm{~cm}$ langen gewundenen Glassäule mit einem inneren Durchmesser von $4 \mathrm{~mm}$. Der Trägergasstrom beträgt $40 \mathrm{~m} l \mathrm{~N}_{2}$ pro Min. Um Druckschwankungen aufzufangen, wird ein hoher Druckgradient zwischen zuführender Gasleitung und Säule durch ein eingebautes Nadelventil aufrecht erhalten. Die Ofentemperatur ist auf $180^{\circ}$ eingestellt, der Injektor wird bei $200^{\circ}$ geheizt, ebenso der FID. Die $\mathrm{H}_{2}$-Flußrate beträgt $40 \mathrm{~m} l$ pro Min., die Luftzufuhr $400 \mathrm{ml}$. Alle Flußraten werden mit einem Seifenfilmströmungsmesser kontrolliert. Die Registrierung der Banden erfolgt über einen Honeywell-Kompensationsschreiber Nr. 561. Die Proben werden mit einer $10 \mu l$ Hamilton-Spritze durch eine Silikonkautschukmembran in den mit Quarzwolle gefüllten Injektor gegeben, Wir erneuern die Quarzwolle nach jeweils etwa 40 Proben,

\section{Trennmaterial}

Von mebreren geprüften Säulenfüllungen erwies sich eine 10proz. Beladung von FFAP') auf Chromosorb WAW 80-100 mesh (beide Fa. Varian Aerograph) als am geeignetsten. Gegenüber anderen stationären Phasen, besonders den sehr gebräuchlichen Polyestern (9-14) ist FFAP aus folgenden Gründen für die Essigsäurebestimmung besonders geeignet:

1. Die Abdampfrate dieser Phase ist selbst bei Gabe von angesäuerten wäßr. Lösungen gering. Dies bedingt bis in den Bereich hoher Verstärkungen hinein eine ruhige Nullinie.

2. Es tritt nur ein getinger sogenannter. Wassergipfel auf. Die Essigsäurebande erscheint trotz ihrer kurzen Retentionszeit davon vollständig getrennt. Störungen durch Aufschulterung werden nicht beobachtet (Abb. 1).

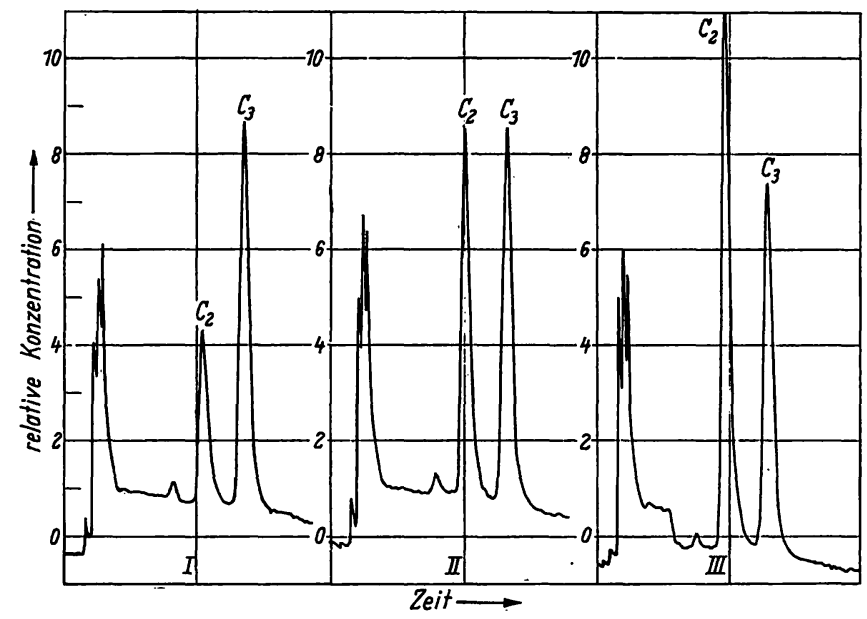

Abb. 1

Originalwiedergabe einer gaschromatographischen Analyse von Essigsäurestandards. $C_{2}=$ Essigsäurebande, $C_{3}=$ Propionsäururebande $\mathrm{I}=50 \mathrm{nMol}, \quad$ II $=100 \mathrm{nMol}$, III $=150 \mathrm{nMol}$ Essigsäure. Propionsäurestandard gleichbleibend $50 \mathrm{nMol}$

3. Die Banden sind weitgehend symmetrisch und schweifen kaum. 4. Erscheinungen, die als "ghosting “ oder "memory effect" beschrieben sind $(8,13,14,15)$, wirken sich nur in geringem $\mathrm{Maße}$ aus.

Die Imprägnierung des Trägermaterials erfolgte nach der von HorNING und Mitarbeitern (16) angegebenen Methode. Als Lösungsmittel für FFAP erwies sich $\mathrm{N}, \mathrm{N}$-Dimethylformamid (Merck) als am geeignetsten. Unter den von uns gewählten Bedingungen ergab sich eine etwa 10proz. Beladung mit der stationären Phase. Nach dem Imprägnieren wurde das Trennmaterial einen Tag an der Luft und weitere $24 \mathrm{Stdn}$. im Wärmeschrank bei $110^{\circ}$ getrocknet.

Für die gefüllten Säulen ließ sich nach

$$
\mathrm{n}=5,54\left(\frac{\mathrm{t}_{\mathrm{dr}}}{\mathrm{b}_{1} / 2}\right)^{2} \text { (15) } \begin{gathered}
\mathrm{t}_{\mathrm{dr}}=\text { Gesamtretentionszeit } \\
\mathrm{b}_{1} / 2
\end{gathered}
$$

eine Trennstufenzahl $n=1600$ errechnen. Bezieht man diese Größe auf die Șäulenlänge $L=1,60 \mathrm{~m}$, so erhält man ein vergleichbares $\mathrm{Maß}$ für die Trennleistung, die sich hier nach

$$
\mathrm{n}^{1}=\frac{\mathrm{n}}{\mathrm{L}}(15)
$$

mit $\mathrm{n}^{1}=1000$ ergibt. Diese Trennleistung ist für unsere Trennaufgabe ausreichend.

Prinzip der Auswertung

Die für die quantitative Auswertung der Analysen gebräuchlichste Methode der Flächenmessung - angenähert ermittelt aus

1) Abkürzung: FFAP bedeutet Free Fatty Acid Phase und stellt ein Reaktionsprodukt zwischen Carbowax $20 \mathrm{M}=$ Polyäthylenglycol und 2-Nitro-Terephthalsäure dar (8). 
Höhe mal Breite in halber Höhe (15) - liefert für die Bestimmung sehr kleiner Essigsäurekonzentrationen wegen des unvermeidlichen Fehlers von Probengabe zu Probengabe keine ausreichend reproduzierbaren Resultate. Dies wurde durch Mitführen eines Inneren Standards korrigicrt. Wir benutzen dazu Propionsäure, die der Lösung, mit der die eingedampften Proben wiederaufgenommen werden, in einer Endkonzentration von $0,5 \mathrm{~mm}$ zugesetzt wird. Zur Eichung der Methode werden die Quotienten der Bandenhöhen eingestellter Acetatstandardlösungen und Innerem Standard gebildet und gegen die Acetatkonzentrationen aufgetragen. Hicraus ergeben sich Eichgraden, aus denen die gesuchte Acetatkonzentration abgelesen werden kann. Streng genommen sind es die Bandenflächen von Analysensubstanz und Standard, die aufeinander bezogen werden müßten. $\mathrm{Da}$ jedoch in unseren Versuchen die Flächen der Standardbande um nicht mehr als max. $15 \%$ voneinander abweichen, können statt dessen die Höhen eingesetzt werden, was dic Auswertung vereinfacht (15).

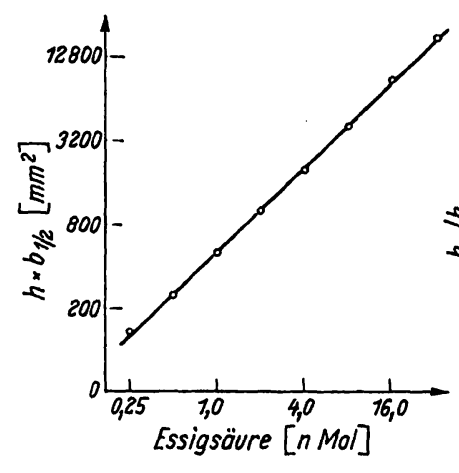

Abb. 2

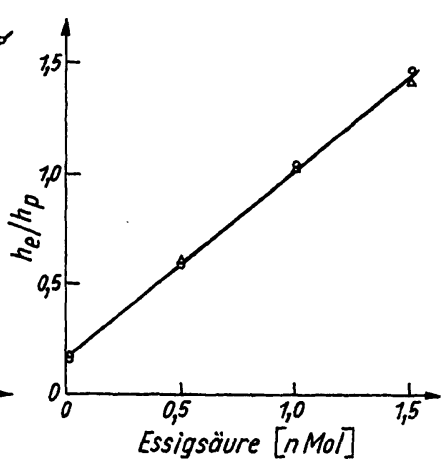

Abb. 3
Abb. 2

Proportionalität zwischen steigenden Essigsäuremengen und Antwort des Gaschromatographen, die sich hier aus der Bandenfläche (angenähert Höhe $\times$ Breite in halber Höhe $\left.\left[h \times b_{1} / 2\right]\right)$ ergibt. Die Koordinaten sind logarithmisch unterteilt

\section{Abb. 3}

Eichkurve nach Vorbehandlung und Gaschromatographie von Essigsäurestandards. Auswertung mit Propionsäure als Innerem Standard. Es sind die absoluten Essịgsäuremengen. gegen den Quotienten der Bandenhöhen von Essigsäure und Propionsäure $\left(\frac{h_{e}}{h_{p}}\right)$ aufgetragen

\section{Ergebnisse und Diskussion}

Wie Abbildung 2 zeigt, antwortet der Gaschromatograph auf die Applikation steigender Essigsäuremengen über einen Bereich von 0,125-32 nMol $/ \mu l$ direkt proportional. In der Eichkurve sind die Bandenflächen gegen die Konzentrationen des Essigsäurestandards aufgetragen.

Zur Prüfung der Reproduzierbarkeit wurde 10mal hintereinander $1 \mu l$ einer $1 \mathrm{~mm}$ angesäuerten Acetateichlösung gespritzt, der wie oben angegeben Propionsäure' in $0,5 \mathrm{~mm}$ Konzentration zugegeben war. Bei Auswertung an Hand des Inneren Standards streuten die Werte um 1,4\%. Das bedeutet eine gute Korrektur der durch ungleichmäßige Probengabe bedingten Schwankungen der $\mathrm{C}_{2}$-Bandenhöhe (Tab. 1). $\mathrm{Da}$ die Ausbeute an Essigsäure bei der Vorbehandlung nicht vollständig ist (vgl. Tab. 2), führten wir regelmäßig Acetatkontrollwerte über den gesamten Analysengang mit. Abbildung 3 gibt eine typische Eichkurve wieder. Der aus der Abbildung ersichtliche Essigsäureblindwert wurde mit ziemlicher Konstanz und regelmäßig beobachtet. Nachdem eine Acetatverunreinigung der verwendeten Reagenzien ausge- schlossen werden konnte, muß eine Verunreinigung durch die in der Laborluft ubiquitär vorkommenden Essigsäurespuren angenommen werden.

Tab. 1

Reproduzierbarkeit der gaschromatographischen Antwort bei 10 maligem Spritzen von $1 \mu l 1 \mathrm{mM}$ Essigsäure. Auswertung mit Propionsäure als Innerem Standard

\begin{tabular}{|c|c|c|c|}
\hline $\begin{array}{c}\text { Probengabe } \\
\mathrm{Nr} .\end{array}$ & $\begin{array}{l}\text { Bandenhöhe } \\
\text { Essigsäure } h_{e}\end{array}$ & $\begin{array}{c}\text { Bandenhöhe } \\
\text { Propionsäure } h_{p}\end{array}$ & $\frac{h_{e}}{h_{p}}$ \\
\hline \multirow[t]{2}{*}{$\begin{array}{r}1 \\
2 \\
3 \\
4 \\
5 \\
6 \\
7 \\
8 \\
9 \\
10\end{array}$} & $\begin{array}{l}58 \mathrm{~mm} \\
61 \mathrm{~mm} \\
58 \mathrm{~mm} \\
63 \mathrm{~mm} \\
64 \mathrm{~mm} \\
64 \mathrm{~mm} \\
65 \mathrm{~mm} \\
67 \mathrm{~mm} \\
68 \mathrm{~mm} \\
65 \mathrm{~mm}\end{array}$ & $\begin{array}{l}56 \mathrm{~mm} \\
61 \mathrm{~mm} \\
57 \mathrm{~mm} \\
63 \mathrm{~mm} \\
64 \mathrm{~mm} \\
64 \mathrm{~mm} \\
65 \mathrm{~mm} \\
67 \mathrm{~mm} \\
68 \mathrm{~mm} \\
65 \mathrm{~mm}\end{array}$ & $\begin{array}{l}1,04 \\
1,0 \\
1,02 \\
1,0 \\
1,0 \\
1,0 \\
1,0 \\
1,0 \\
1,0 \\
1,0\end{array}$ \\
\hline & $\begin{array}{l}\text { Mittelwert } \\
\text { Standardabweic } \\
\text { Variationskoeffi }\end{array}$ & $\begin{aligned} \bar{x} & =1,006 \\
\text { ing } & = \pm 0,014 \\
\text { ent } V K & =1,4\end{aligned}$ & \\
\hline
\end{tabular}

Tab. 2

Wiederfindeversuche mit eingesetzten bekannten Acetatmengen. In der letzten Spalte sind die wiedergefundenen ${ }^{14} \mathrm{C}$-Acetatmengen der letzten Spalte sind die wiedergefund

\begin{tabular}{ccccc}
\hline $\begin{array}{c}\text { Versuch } \\
\text { Nr. }\end{array}$ & $\begin{array}{c}\text { eingesetzt } \\
\text { nMol }\end{array}$ & nMol & $\begin{array}{c}\text { wiedergefunden } \\
\%(\bar{x})\end{array}$ & $\%{ }^{\text {w. C }}(\overline{\mathbf{x}})$ \\
\hline 1 & 100 & 84 & 84 & - \\
2 & $50 / 100 / 150$ & $45 / 86 / 129$ & 87 & 85 \\
3 & $50 / 100 / 150$ & $45 / 81 / 129$ & 86 & 87 \\
4 & $50 / 100 / 150$ & $49 / 89 / 135$ & 86 & 81 \\
5 & $50 / 100 / 150$ & $39 / 86 / 93$ & 93 & - \\
6 & $50 / 100$ & $46 / 93$ & & \\
\hline
\end{tabular}

Um zu erfahren, wieviel Essigsäure bei der Vorbehandlung der Proben verlorengeht, führten wir Vergleichsbestimmungen nicht vorbehandelter und behandelter Acetatstandards durch. Wie aus Tabelle 2 ersichtlich, werden hierbei im Durchschnitt $87 \%$ des eingesetzten Acetats wiedergefunden. Kontrollversuche mit ${ }^{14} \mathrm{C}$ markiertem Acetat bestätigten diese Ergebnisse. Sie zeigten darüber hinaus, daß der geringere Teil der Essigsäure während der Mikrodestillation verlorengeht und der Hauptverlust während des Eindampfens und Wiederaufnahme der Proben auftritt. $\mathrm{Da}$ die Auswertung der Meßergebnisse - wie schon erwähnt an Hand mitgeführter Standardwerte erfolgt, bleibt dieser Verlust ohne Auswirkung.

Einen weiteren Hinweis für die Zuverlässigkeit der Methode erhielten wir schließlich durch Bestimmung des Acetatgehaltes verschieden verdünnter Plasmaproben. Dabei verhielten sich die gefundenen Werte den Verdünnungen entsprechend.

Tab. 3

Acetatspiegel in menschlichem Plasma, Harn und Liquor cerebrospinalis aus einem nicht ausgewählten Patientengut einer Inneren Abteilung und einer Kinderklinik

\begin{tabular}{|c|c|c|c|c|c|}
\hline $\begin{array}{l}\text { Plasma- } \\
\text { probe } \\
\text { Nr. }\end{array}$ & $\begin{array}{c}\text { Acetat } \\
\mathrm{nMol} / \mathrm{ml}\end{array}$ & $\begin{array}{c}\text { Harnprobe } \\
\text { Nr. }\end{array}$ & $\begin{array}{c}\text { Acetat } \\
\mathrm{nMol} / \mathrm{m} l\end{array}$ & $\begin{array}{c}\text { Liquor- } \\
\text { probe } \\
\text { Nr. }\end{array}$ & $\begin{array}{l}\text { Acetat } \\
\mathrm{nMol} / \mathrm{ml}\end{array}$ \\
\hline $\begin{array}{l}31 \\
32 \\
33 \\
34 \\
35 \\
36 \\
37 \\
38 \\
39 \\
40\end{array}$ & $\begin{array}{l}90,0 \\
15,5 \\
24,5 \\
22,5 \\
42,5 \\
45,5 \\
27,5 \\
45,5 \\
48,5 \\
39,0\end{array}$ & $\begin{array}{r}1 \\
2 \\
3 \\
4 \\
5 \\
6 \\
7 \\
8 \\
9 \\
10\end{array}$ & $\begin{array}{l}23,5 \\
35,5 \\
25,0 \\
19,5 \\
29,5 \\
16,0 \\
21,5 \\
38,5 \\
30,5 \\
10,5\end{array}$ & $\begin{array}{l}21 \\
22 \\
23 \\
24 \\
25 \\
26 \\
27 \\
= \\
=\end{array}$ & $\begin{array}{r}6,0 \\
76,0 \\
17,0 \\
31,0 \\
59,0 \\
39,0 \\
75,0 \\
= \\
=\end{array}$ \\
\hline
\end{tabular}


Acetatbestimmung in verschiedenen Körperflüssigkeiten

Als Anwendungsbeispiel der Methode sind in Tabelle 3 die Ergebnisse von Acetatbestimmungen in Plasmaund Harnproben sowie im Liquor cerebrospinalis wiedergegeben. Vergleicht man die Mittelwerte, so ergibt sich ein recht großer Streubereich. Die Mittelwerte liegen bei $40,1 \mathrm{nMol} / \mathrm{m} l$ Plasma $(s= \pm 20,9), 25,0 \mathrm{nMol} /$ $\mathrm{m} l$ Harn $(\mathrm{s}= \pm 8,7)$ und $42,9 \mathrm{nMol} / \mathrm{m} l$ Liquor $(\mathrm{s}=$ 27,7). Bisher sind beim Menschen lediglich Plasmaacetatspiegel enzymatisch gemessen worden $(17,18)$. Sie liegen mit Werten $z$ wischen $50-100 \mathrm{nMol} / \mathrm{m} l$ etwas höher als die hier gaschromatographisch gefundenen. Im Vergleich zu den langkettigen unveresterten Plasmafettsäuren macht die freie Essigsäure demnach etwa den zehnten Teil aus. Vergleichbare Bestimmungen des Harn- und Liquoracetats liegen in der Literatur nicht vor.

Auf Grund unserer Erfahrungen können wir die hier beschriebene gaschromatographische Methode zur Acetatbestimmung gegenüber dem enzymatischen Sulfanil-
amid-Acetylierungstest sowohl im Hinblick auf die einfachere Handhabung als auch hinsichtlich der Reproduzierbarkeit der Ergebnisse als einen eindeutigen Fortschritt ansehen. Eine weitere Vereinfachung durch Verzicht auf die vorausgehende Abtrennung der Essigsäure durch Mikrodestillation, wie von anderer Seite beschrieben wurde $(4,14)$, erscheint uns nicht zweckmäßig. Bei direktem Einsatz von enteiweißten und eingeengten Proben werden nämlich im biologischen Material vorkommende höhere Homologen unter unseren Bedingungen mitaufgezeichnet, was bei den zum Teil erheblichen Retentionszeiten einen wesentlich größeren Zeitaufwand pro Analyse bedeuten würde. Die durch Auslassen der Mikrodestillation erreichte Zeitersparnis ginge damit wieder verloren. Hinzu kommt, daß der Injektor durch nicht flüchtige Bestandteile rasch verunreinigt wird.

Die Arbeit wurde mit Unterstützung der Deutschen Forschungsgemeinschaft Bad Godesberg (Schwerpunktprogramm „Ernährungsforschung ") durchgeführt. Für wertvolle methodische Hinweise danken wir Herrn Dr. B. Hampreckir und Herrn Dr. R. SeIfert am Max Planck Institut fụ̈r Zellechemie, Münçhen.

\title{
Literatur
}

1. Lundeuist, F., U. Fugmann und H. Rasmussen, Biochem. J., 80, 393 (1961). - 2. Conway, E. J., Mikrodiffusion Analysis, 4. Aufl., Crosby, Lockwood and Son I.td., London (1957). 3. Bergmeyer, H. U. und H. Moellering, Biochem. Z., 344, 167 (1966). - 4. MEdzIHRAdsky, F. und W. LAMPrecht, HoppeSeylers Z. physiol. Chem., 343, 35 (1965). - 5. BARtLEX, W., Biochem. J., 53, 305 (1953). - 6. Boetrger, I., U. Deutrcke, E. Prüsse-Evertz, B. D. Ross und O. Wreland, Zschr. exper. Med., 145, 346 (1968).-7.HochHeuser, H., H. WeIsS und O. WIELAND, diese Z. 6, 175 (1964). - 8. BAKER, R. A., J. Gas Chromatog., 4, 418 (1966). - 9. Appleby, A. J. und J. E. O. MAYNE, J. Gas Chromatog., 5, 211 (1967). - 10. Kaplanoví, B. und J.
JanÁx, Mikrochịm. Acta, 1-2, 119 (1966). - 11. Lough, A. K., R. S. Reid, M. Murray und F. M. Black, J. Sci. Food Agric., 18, 214 (1967). - 12. Kung, J. T., R. P. Naught und J. A. YERANSian, J. Food Sci., 32, 455 (1967). - 13. SallmanN, H. P. und K. H. Niesar, Z. Tierphysiol. Tierern., 23, 6 (1967). - 14. Baumgardr, B. R., Departmental Bulletin 1, Dptm. Dairy Science, Univ. of Wisconsin, Madison (1964). - 15. KArser, R., Chromatographie in der Gasphase, Bibliographisches Institut Mannheim (1962). - 16. Hokning, E. C., E. A. Moscatelit und C. C. Sweelex, Chem. and Ind., 1959, 751. - 17. LundQuist, F., Acta physiol. Scand., 50, 175 (1960). - 18. Werss, H., Dissertation München (1965).

\section{Fluorometrische Bestimmung von Testosteron im menschlichen Plasma}

\author{
Von V. Graef und HJ. Staudinger \\ Aus dem Physiologisch-Chemischen Institut der Universität Gießen (Direktor: Prof. Dr. Hj. Staudinger)
}

. (Eingegangen am 29. März 1968)

Herrn Prof. Dr. med. Dr. rer. nat. Ernst Schütte qum 60. Geburtstag gewidmet

Es wird eine Methode zur Bestimmung von Testosteron im Plasma des Menschen beschrieben. Bei dieser Methode wird Testosteron durch Dünnschichtchromatographie abgetrennt. Die Fluoreszenz-Reaktion auf einer Oberfläche von Lithiumhydiroxid dient zur quantitativen Bestimmung. Durch diese Methode kann noch 0,01 $\mu \mathrm{g}$ Testosteron bestimmt werden.

A method for the determination of testosterone in human plasma is described. Testosterone is separated by thin-layer chromatography, and determined quantitatively by the fluorescence reaction on a surface of lithium hydroxide. As little as $0.01 \mu \mathrm{g}$ testosterone can be determined by the above method.

Kürzlich berichteten wir über eine Methode zur Bestimmung von Testosteron im Harn des Menschen, die darauf beruht, daß Testosteron auf der Oberfläche eines
Lithiumhydroxid-Preßlings eine Fluoreszenz gibt (1). $\mathrm{Da}$ diese Fluoreszenzreaktion spezifisch für alle $\Delta^{4}-3$ Ketosteroide ist, mußte das Testosteron vorher von 